Instructions for authors, subscriptions and further details:

http://qre.hipatiapress.com

\title{
Participación de Familias en Educación Física
}

José Manuel Castanedo ${ }^{1} \&$ Marta Capllonch ${ }^{1}$

1) Facultad de Educación, Universidad de Barcelona, Spain.

Date of publication: October $28^{\text {th }}, 2018$

Edition period: October 2018 - February 2019

To cite this article: Castanedo, J. M., \& Capllonch, M. (2018).

Participación de familias en Educación Física. Qualitative Research in

Education, 7(3), 304-334. doi:10.17583/qre.2018.3603

To link this article: $h t t p: / / d x . d o i . o r g / 10.17583 / q r e .2018 .3603$

\section{PLEASE SCROLL DOWN FOR ARTICLE}

The terms and conditions of use are related to the Open Journal System and to Creative Commons Attribution License (CC-BY). 


\section{Families Involvement in Physical Education}

José Manuel Castanedo

Universidad de Barcelona
Marta Capllonch

Universidad de Barcelona

(Received: 26 June 2018; Accepted: 22 October 2018; Published: 28 October 2018)

\section{Abstract}

The International Scientific Community identifies the involvement of families in school as a Successful Educational Actions, whose strict implementation leads to the improvement in the academic achievement as well as in the coexistence in the educational context. The aim of this investigation, conducted under the approach of the critical communicative methodology, is to prove how the involvement of the families in education and assessment in the area of Physical Education within the framework of the pedagogical model "Individual Challenges with Shared Responsibility" means an enhancement in all students and their context. The outcomes show an improvement in the academic performance and in the environmental education of students, including those with the greatest barriers. Likewise, this study brings to light the transfer of learning to other contexts and the transformation of the school-family relationship. Besides, it provides evidence on the benefits that it brings to the families that have participated. This study opens up new lines of research into the overcoming of the limitations detected and the improvement of the educational, family and even community contexts.

Keywords: success educational actions, Physical Education, family involvement, pedagogical model 


\section{Participación de Familias en Educación Física}

José Manuel Castanedo

Universidad de Barcelona
Marta Capllonch

Universidad de Barcelona

(Recibido: 26 de junio de 2018; Aceptado: 22 de octubre de 2018; Publicado: 28 de octubre de 2018)

\section{Resumen}

La Comunidad Científica Internacional identifica la participación de las familias en la escuela como una Actuación Educativa de Éxito, conduciendo su implementación rigurosa a mejoras en los resultados académicos y convivenciales en el contexto educativo. El objetivo de esta investigación, bajo el enfoque de la metodología comunicativa crítica, es evidenciar cómo la participación educativa y evaluativa de familiares en el área de Educación Física dentro del marco del modelo pedagógico "Retos Individuales con Responsabilidad Compartida" produce mejoras en todo el alumnado y su entorno. Los resultados demuestran la mejora curricular y convivencial del alumnado, incluido aquel con mayores dificultades. Se destaca, así mismo, la transferencia de los aprendizajes a otros contextos y la transformación de la relación escuela-familia. También se evidencian contribuciones positivas para las familias participantes. Este estudio abre nuevas líneas de investigación para superar las limitaciones detectadas y para mejorar los contextos educativo, familiar e incluso comunitario.

Palabras clave: actuaciones educativas de éxito, Educación Física, modelo pedagógico, participación de familiares. 


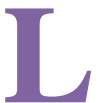

a creencia de que el papel educativo de las familias se desarrolla principalmente en el hogar se encuentra muy extendida entre el profesorado a pesar del creciente deseo de las familias por implicarse educativamente en las escuelas y en las aulas (Gokturk \& Dinckal, 2017). En el área de Educación Física (EF) ese deseo es fundamental para fomentar una EF de calidad, apoyar programas de salud o conseguir incrementos de práctica física (Erwin, Beets, Centeio, \& Morrow, 2014; Kehm, Davey, \& Nanney, 2015; Michael, Dittus, \& Epstein, 2007; Wilhelmsen \& Sørensen, 2018). El presente trabajo de investigación analiza la participación educativa de las familias en las sesiones de EF a través de un modelo pedagógico inclusivo denominado Retos Individuales con Responsabilidad Compartida (RIRC).

\section{Marco Teórico}

Existen numerosos proyectos educativos que cuentan con la participación comunitaria para mejorar los aprendizajes del alumnado: School Development Program, Accelerated Schools, Education for All, Escuelas Democráticas, Comunidades de Aprendizaje. No obstante, la investigación demuestra que aparte de estos, casi cualquier escuela con éxito cuenta con la participación de familiares como eje clave en su proyecto educativo (Apple \& Beane, 2012). La literatura científica evidencia que la participación familiar y comunitaria contribuye no solo a mejorar el bienestar en las escuelas, sino a incrementar también los resultados de aprendizaje de los niños y niñas (Caspe, Lopez, \& Wolos, 2007; Dearing, Kreider, Simkins, \& Weiss, 2006; Driessen, Smit, \& Sleegers, 2005; Fan \& Chen, 2001; Garbacz, Zerr, Dishion, Seeley, \& Stormshak, 2018; Hampden-Thompson, \& Galindo, 2017; Henderson \& Mapp, 2002; Hill \& Taylor, 2004; Ma, Shen, Krenn, Hu, \& Yuan, 2016; Pomerantz, Grolnick, \& Price, 2005; Senechal \& LeFevre 2002).

Sin embargo, no todos los tipos de participación tienen una relación consistente y positiva con el rendimiento académico del alumnado (Boonk, Gijselaers, Ritzen, \& Brand-Gruwel, 2018). De las investigaciones que se han centrado en identificar los modelos más efectivos de participación familiar y comunitaria, destaca el INCLUD-ED Project (2006-2011), reconocido como la investigación de mayor rango científico y con mayores recursos existente en Europa sobre educación escolar (Flecha, García, 
Gómez, \& Latorre, 2009). De los cinco modelos de participación familiar y comunitaria que se identificaron en la investigación INCLUD-ED Consortium (2011) -informativa, consultiva, decisoria, evaluativa y educativa-, son los tres últimos los que influyen más directamente en el éxito académico y la mejora de la convivencia en los centros educativos (Martínez \& Niemelä, 2010). La participación decisoria incluye las voces de familiares en los procesos de toma de decisiones, en la gestión y en la organización del centro. En la participación evaluativa, las familias están involucradas en la evaluación de los aprendizajes y resultados del estudiantado, así como en los resultados de la escuela. Por último, la participación educativa, que ha demostrado tener el mayor impacto positivo en los resultados de aprendizaje (INCLUD-ED Project, 2006-2011), incluye a las familias y otros miembros de la comunidad en las actividades curriculares y extracurriculares que se implementan tanto dentro como fuera del aula, así como la propia formación de familiares.

La participación de las familias de manera sincera y honesta en los procesos de toma de decisión, en el currículum, en la evaluación, en las aulas y en espacios de aprendizaje ha transformado contextos educativos de gran complejidad en escuelas más exitosas (Alexiu \& Sordé, 2011; Bonell \& Ríos, 2014; Díez, Gatt, \& Racionero, 2011; Flecha, 2012; Flecha \& Soler, 2013; Flecha et al., 2009; Gatt, Ojala, \& Soler, 2011; Girbés, Macías, \& Álvarez., 2015; Tellado \& Sava, 2010). Este éxito escolar se concreta en la reducción del absentismo, la superación y/o disminución de desigualdades y de situaciones de exclusión, la mejora y aumento de los aprendizajes instrumentales y competenciales de todo el alumnado, el fomento de la solidaridad y la reducción de conflictos y mejora de la convivencia escolar.

INCLUD-ED Project (2006-2011) identificó así un tipo de actuaciones universales y transferibles denominadas Actuaciones Educativas de Éxito (AEE), en las que se incluye la participación voluntaria y solidaria de familias y otras personas de la comunidad: Interactive Groups, Extending Learning Time y Dialogic Reading. Aunque la implementación de AEE proporciona a todos los niños y niñas no sólo las mismas oportunidades de aprendizaje sino las condiciones para la igualdad de resultados (Flecha, 2011), la participación de familiares y de la comunidad se puede concretar en otras actuaciones diferentes. Este trabajo aporta a la comunidad una nueva actuación que conjuga la participación educativa y evaluativa de 
familiares con el aprendizaje dialógico (ApD) (Aubert, Flecha, García, Flecha, \& Racionero 2008; Flecha, 2012; Flecha, 1997; Racionero, Ortega, García, \& Flecha, 2012) dentro del modelo pedagógico emergente RIRC contextualizado en el área de EF.

El ApD es una concepción del aprendizaje cuya "principal característica (...) es la interacción y la comunicación como factores clave del aprendizaje" (Aubert et al., 2008, p. 24). El ApD, junto a los principios de la educación inclusiva -presencia, participación y aprendizaje o logro(Booth \& Ainscow, 2015), fundamentan el modelo RIRC. En su estructura didáctica, la participación de familiares y de personas de la comunidad es un elemento clave para avanzar hacia la igualdad de resultados del alumnado (Aubert et al., 2008) a través de interacciones basadas en el diálogo igualitario (Racionero, 2010), una enseñanza dirigida a máximos, intereses compartidos, fomento de la autonomía, evaluaciones dialógicas (Castanedo \& Capllonch, 2017), altos niveles de apoyo al alumnado e información continuada del proceso educativo.

En el modelo RIRC, la estructura de las sesiones, la distribución de los contenidos en forma de retos, la explicación de los mismos, la temporalización y la puntuación asignada a cada reto debe estar visible y accesible para facilitar que tanto el alumnado como cualquier persona participante sepa en todo momento qué hacer, cuándo, cuánto tiempo, cómo y con quién (Tabla 1). 
Tabla 1

Ejemplo de información visible para el alumnado de $6^{\circ}$ curso

Estructura de la sesión y distribución de los retos del tercer trimestre

\begin{tabular}{|c|c|c|}
\hline Sesión 1 (lunes; 45’) & Sesión 2 (martes; 60’) & Sesión 3 (viernes; 45’) \\
\hline \multirow{2}{*}{$\begin{array}{l}12^{\prime} \text { Reto } 1 \text { juegos } \\
\text { (marrow) }\end{array}$} & $10^{\prime}$ Reto 2 de juegos & $0^{\prime}$ Reto zancos \\
\hline & $\begin{array}{l}\text { 10’ Reto } 3 \text { lanzamiento } \\
\text { (béisbol) }\end{array}$ & 10' Reto 4 lanzamientos \\
\hline \multirow[t]{2}{*}{$\begin{array}{l}12^{\prime} \text { Reto } 1 \text { lanzamientos } \\
\text { (red) }\end{array}$} & $\begin{array}{l}10^{\prime} \text { Reto } 2 \text { lanzamiento } \\
\text { (frisbee) }\end{array}$ & $\begin{array}{l}8^{\prime} \text { Reto } 1 \text { puntería (b } \\
\text { 10’ Reto } 2 \text { puntería } \\
\text { (dardos) }\end{array}$ \\
\hline & $10^{\prime}$ Reto peonza & \\
\hline \multirow[t]{2}{*}{$\begin{array}{l}\text { Evaluación formativa y } \\
\text { compartida }\end{array}$} & $10^{\prime}$ Reto bádminton & $\begin{array}{l}\text { Evaluación formativa y } \\
\text { compartida }\end{array}$ \\
\hline & $\begin{array}{l}\text { Evaluación formativa y } \\
\text { compartida }\end{array}$ & \\
\hline
\end{tabular}

\section{Explicación de los retos y puntuación}

Reto 1 de juegos (marrow): hacer un "marrow" ( 0,8 puntos)

Reto de bádminton: Dominio del lob y del clear de derechas y de revés $(0,6$ puntos)

Reto 1 lanzamientos (red): 3 intercambios de balones utilizando dos redes, una por pareja (0,6puntos) 수숭

Reto 2 de juegos (cazacaballos): Pillar a 10 caballos cuando se es pillador y realizar 5 viajes y 5 salvados cuando se es caballo. ( 0,8 puntos)

Reto 3 de lanzamiento (béisbol): lanzar y batear correctamente (0,6puntos)

Reto 2 de lanzamiento (frisbee): pases desde cerca intercambiando 2 discos $(0,6$ puntos)

in

Reto de peonza: bailar la peonza demostrando dominio con 3 trucos (0,6puntos)

Reto de zancos: andar con zancos y realizar 3 trucos (0,6puntos)

Reto 4 lanzamientos (pasabolo tablón): derribar 3 bolos en un turno (0,6puntos)

果

Reto 1 de puntería (botes): derribar 5 botes con 2 intentos (0,6puntos)

Reto 2 de puntería (dardos): clavar un dardo en la cantidad indicada antes de tirar (0,6puntos) 


\section{Castanedo \& Capllonch-Families involvement in P. E.}

Los retos son variados en relación a los contenidos curriculares, siendo su superación un indicador de logro de los estándares de aprendizaje vinculados a la actividad del reto. A lo largo del bimestre o del trimestre, cuando un alumno o alumna demuestra el dominio de un reto, lo registra en una hoja de control y a partir de ahí se abren dos opciones no excluyentes que escoge de manera voluntaria. Una opción es seguir trabajando en un reto mejorado que cumpla el criterio de compartir la misma naturaleza que el reto original pero con dificultades añadidas evidentes. Otra opción es ayudar consciente y solidariamente a un compañero o compañera a mejorar o superar el reto en el que esté trabajando o aquel en el que encuentre mayores dificultades para su consecución. Al final de cada sesión existe un tiempo para la evaluación dialogada. En base a unos códigos establecidos (Castanedo \& Capllonch, 2017), cada alumno y cada alumna valora su trabajo de manera compartida y dialogada con el resto del alumnado, familiares y docente, quienes pueden apoyar o rebatir las valoraciones individuales en condiciones de diálogo igualitario y argumentos de validez.

En el modelo RIRC se crean los entornos de aprendizaje necesarios para fomentar interacciones entre iguales, con iguales más capaces, con iguales de cursos diferentes, con familiares y con miembros de la comunidad, posibilitando oportunidades de aprendizaje diversas. En definitiva, facilita lo que Vygotsky ya indicaba cuando afirmaba que "el aprendizaje activa una serie de procesos internos de desarrollo que son capaces de operar solo cuando el niño [o la niña] está interactuando con personas de su entorno y en cooperación con sus compañeros" (1979, p. 89). Idea que desde el ApD se sigue apoyando al considerar que las interacciones generadoras de aprendizaje ocurren entre pares pero también entre niños, niñas y personas adultas de referencia, incluyendo profesorado, familiares y otros miembros de la comunidad (García, 2012).

Esta investigación aporta evidencias de que la participación educativa y evaluativa de familiares mejora los resultados curriculares y convivenciales del alumnado, centrándose el estudio específicamente en el área de EF y dentro del marco del modelo pedagógico inclusivo RIRC. 


\section{Método}

\section{Metodología}

Se ha seguido el enfoque metodológico comunicativo crítico (Gómez, Latorre, Sánchez, \& Flecha, 2006), combinando el conocimiento científico con las voces de las personas participantes en la investigación (Gómez, Puigvert, \& Flecha, 2011; Gómez, Racionero, \& Sordé, 2010; Puigvert, Christou, \& Holford, 2012) para construir de manera conjunta un nuevo conocimiento a través de actos comunicativos dialógicos que minimizan las relaciones de poder que suelen producirse en los procesos comunicativos (Sordé \& Ojala, 2010) y de investigación. Esta metodología busca validar junto con las personas participantes los actos, fenómenos, interacciones, ideas, reflexiones, aportaciones y hechos relacionados con la realidad investigada, prestando especial atención a los elementos que generan exclusión y a aquellos que pueden transformar y mejorar la realidad de partida.

La creación de conocimiento compartido implica entendimiento y es útil, no por su adquisición, sino por el uso que se hace de él. Para conseguirlo, se establecen relaciones dialógicas entre quienes investigan y las personas investigadas guiadas por la reflexión, la autorreflexión y la intersubjetividad. Con el fin de asegurar el rigor de la investigación (Linconl \& Guba, 1985) y de eliminar sesgos, se crea un consejo asesor formado por personas de los diferentes colectivos que participan en la investigación (tabla 2) cuyo diálogo, reflexión y feedback en momentos clave de la investigación, permite contrastar el conocimiento científico con los resultados de la investigación y las experiencias y vivencias de las personas participantes. 
312 Castanedo \& Capllonch-Families involvement in P. E.

Tabla 2

Participantes del consejo asesor

Participante

Relación

\begin{tabular}{ll}
1 & Experta Aprendizaje Dialógico \\
2 & Alumna $5^{\circ}$ \\
3 & Maestra y madre no voluntaria \\
4 & Maestra EF $\left(1^{\circ}\right.$ y EI $)$ \\
5 & Madre voluntaria \\
6 & Investigador y maestro EF $\left(2^{\circ}\right.$ a $\left.6^{\circ}\right)$ \\
\hline
\end{tabular}

\section{Participantes}

La investigación se lleva a cabo durante el curso escolar 2016/2017 con el alumnado de nueve grupos $\left(1^{\circ} \mathrm{A}, 1^{\circ} \mathrm{B}, 2^{\circ} \mathrm{A}, 2^{\circ} \mathrm{B}, 3^{\circ}, 4^{\circ} \mathrm{A}, 4^{\circ} \mathrm{B}, 5^{\circ}\right.$ y $\left.6^{\circ}\right)$ de los seis cursos de educación primaria en un centro público de educación obligatoria de la comunidad autónoma de Cantabria (España) y con la participación de 103 familiares. La participación educativa y evaluativa de las familias se desarrolló en las sesiones de EF dentro del modelo pedagógico RIRC con una temporalización de 4 meses del curso escolar -de febrero a mayo-, registrándose un número total de 350 participaciones durante este periodo. En centro está ubicado en un entorno rural, siendo el turismo la principal fuente de trabajo del municipio.

\section{Procedimiento}

A través de una nota informativa dirigida a todos los padres, madres y tutores o tutoras legales del alumnado, se explicaron los beneficios de la participación educativa de las familias en los centros educativos y se invitaba y animaba a todas las personas interesadas a involucrase en la educación de los niños y niñas participando en el área de EF. En su primera participación, las familias recibían una formación por parte del maestro 
investigador centrada en tres aspectos fundamentalmente: 1) explicación de las funciones a desempeñar: dinamizar y potenciar los diálogos, ofrecer apoyo al alumnado e informar sobre situaciones e interacciones poco igualitarias -relaciones de poder entre compañeros, no respetar decisiones tomadas en grupo, no dejar hablar a compañeros o compañeras que desean dar una opinión, saltarse los turnos establecidos en una actividad, acaparar los mejores materiales y espacios- y cómo intervenir en su caso comunicárselo al profesor y/o hacer visible a los niños y niñas lo que está pasando y establecer un diálogo para buscar una solución; 2) explicación de aspectos organizativos: asistencia voluntaria de cualquier familiar en cualquier sesión y sin necesidad de avisar, libertad en las interacciones y apoyos tanto instrumentales como motivacionales al alumnado a lo largo de toda la sesión; y 3) explicación de la dinámica de trabajo de las sesiones bajo el modelo RIRC: explicación de los principios del aprendizaje dialógico y de la educación inclusiva, organización de las sesiones y de los contenidos, organización de apoyos, interacciones, etc.

Cuando los familiares participan en las sesiones lo que hacen principalmente es apoyar a los alumnos para que consigan los retos, bien animándoles, dándoles consejos, fomentando diálogos que les hagan tomar mejores decisiones, ayudándoles a organizarse mejor, dinamizando interacciones, evitando situaciones injustas, ofreciendo más apoyo a quien tiene más dificultades con algún reto, animando a los niños y niñas a compartir lo que saben con los demás... Los familiares pueden moverse libremente por el espacio e interaccionar libremente con los niños y niñas que deseen.

\section{Técnicas de Recogida de Información}

Todas las técnicas de recogida de información (tabla 3), ya sean instrumentos de naturaleza cuantitativa (registros) o estrategias cualitativas (relatos comunicativos de vida cotidiana, grupos de discusión y observación participante comunicativa), se aplican bajo una orientación comunicativa estableciéndose siempre un diálogo entre las personas investigadas y las personas que investigan. Los diálogos, registrados en notas de campo, registros en vivo con descripciones y reflexiones percibidas, suceden en el contexto natural de las personas participantes: sesiones de EF, al final de las sesiones, en los recreos o en momentos no lectivos establecidos para tal fin. 
314 Castanedo \& Capllonch-Families involvement in P. E.

Durante los cuatro meses de trabajo de participación familiar se recogía la información principalmente mediante la observación participante; una vez finalizado ese periodo se realizaron relatos comunicativos de vida cotidiana a familiares, alumnado y a una maestra de EF. De acuerdo con la metodología comunicativa, las personas participantes se implican en el proceso investigador, participando en la elaboración de las técnicas de recogida de la información a través del consejo asesor -revisando los guiones de los relatos comunicativos, los registros, los guiones del grupo de discusión-, y en la validación de los resultados a través del grupo de discusión. 
Tabla 3

Recogida de información

\begin{tabular}{|c|c|c|c|}
\hline $\begin{array}{l}\text { Técnicas de } \\
\text { recogida de } \\
\text { información }\end{array}$ & Código & Perfil persona participante & Número detécnicas \\
\hline \multirow{3}{*}{$\begin{array}{c}\text { Relatos } \\
\text { comunicativos de } \\
\text { vida cotidiana }\end{array}$} & RA & Alumnado & 15 \\
\hline & $\mathrm{RM}$ & Maestra & 1 \\
\hline & $\mathrm{RF}$ & Familiares & 5 \\
\hline $\begin{array}{l}\text { Grupo de } \\
\text { discusión } \\
\text { comunicativo }\end{array}$ & GD & $\begin{array}{l}\text { Familiares voluntarios, alumna } \\
\text { participante, maestra, experta e } \\
\text { investigador }\end{array}$ & 1 grupo de discusión \\
\hline $\begin{array}{l}\text { Observación } \\
\text { participante } \\
\text { comunicativa }\end{array}$ & $\mathrm{OPc}$ & $\begin{array}{l}\text { Docente como investigador en } \\
\text { el aula }\end{array}$ & $\begin{array}{l}54 \text { (en todos los } \\
\text { espacios y tiempos) }\end{array}$ \\
\hline \multirow[t]{3}{*}{ Registros } & $\mathrm{Rr}$ & Retos superados & 16 \\
\hline & $\mathrm{Rp}$ & Familiares participantes & 1 \\
\hline & $\operatorname{Re}$ & Evaluación diaria y final & 28 \\
\hline Consejo asesor & $\mathrm{CA}$ & $\begin{array}{l}\text { Padre voluntario, alumno } \\
\text { participante, profesor EF, } \\
\text { experta e investigador }\end{array}$ & 1 consejo asesor \\
\hline TOTAL & & & 122 \\
\hline
\end{tabular}

\section{Análisis de la Información}

Toda la información obtenida ha sido analizada, previa transcripción, siguiendo los componentes propios de la metodología comunicativa. En el nivel básico de análisis se agrupó la información de manera inductiva estableciéndose las categorías "aportación de las familias" y "aportación a las familias", con el apoyo del Software NVivo for Mac 11.4, cruzándose con las dimensiones transformadoras (situaciones, hechos o actuaciones en 
los que la participación de las familias contribuye a mejorar los resultados curriculares y convivenciales del alumnado) y con las dimensiones exclusoras (información relativa a situaciones, hechos o actuaciones derivados de la participación de familiares que impiden, dificultan o limitan los resultados curriculares y convivenciales del alumnado). En un nivel amplio de análisis, se establecieron de manera deductiva las siguientes subcategorías (ver tabla 4):

- Apoyo: ayuda consciente dirigida a quienes más lo necesitan.

- Información: conocimiento claro, explicativo y a tiempo dirigido a las familias sobre objetivos, finalidades $y$ funciones de la participación; incluyendo toda la información relativa a aspectos educativos que proviene de diferentes vías (profesorado, alumnado, familiares).

- Participación: presencia activa de familiares que no se limita a la simple presencia.

- Resultados: aprendizaje de las familias y mejora de los aprendizajes y de la convivencia del alumnado como consecuencia de esta participación educativa y evaluativa.

- Transferencia: repercusión de lo ocurrido en las sesiones de EF en contextos diversos (escuela, familia, barrio) y a niveles diferentes: personal, grupal, comunitario.

- Valores y Sentimientos: motivación, relaciones de amistad, seguridad, solidaridad y relación con la escuela

Tabla 4

Sistema de categorización ${ }^{1}$

Aportación de las familias

Aportación a las familias
A. I. P. R. T. Vy
A. I. P. R. T. Vy
$\mathrm{S}$
A. I. P. R. T. $\mathrm{S}$

Dimensiones

$\begin{array}{llllll}1 & 2 & 3 & 4 & 5 & 6\end{array}$

$\begin{array}{llllll}7 & 8 & 9 & 10 & 11 & 12\end{array}$

Transformadoras

Dimensiones

$\begin{array}{llllll}13 & 14 & 15 & 16 & 17 & 18\end{array}$

$\begin{array}{lllll}19 & 20 & 21 & 22 & 23\end{array}$

24

Exclusoras 
El análisis de la información finaliza con la descripción de los datos en un informe de resultados que se presenta al grupo de discusión con el fin contrastarlo con las personas que componen el grupo y así validar la contribución de los resultados obtenidos al contexto educativo y analizar la validez de las interpretaciones realizadas eliminando posibles sesgos.

\section{Discusión y resultados}

El volumen de la información recogida dificulta un análisis detallado de los resultados subcategoría por subcategoría, contando la investigación con 122 registros repartidos en las diferentes técnicas utilizadas, por lo que se presentan los resultados analizados por categorías.

\section{Dimensión Transformadora: Aportación de las Familias. Situaciones, Hechos o Actuaciones en las que la Participación de las Familias Facilita o Posibilita Aprendizajes}

La participación de familiares aumenta el número y la diversidad de oportunidades del alumnado para mejorar sus resultados, ya que incrementa las interacciones comunicativas cargadas de lenguaje, un factor clave para el aprendizaje (Aubert et al., 2008; Mercer, Hargreaves, \& García, 2017). A medida que la asistencia se hace más regular y consciente, los familiares interaccionan más con el alumnado, ayudando más a quien más lo necesita independientemente del parentesco. La estabilidad se identifica en la participación como un factor de mejora en los resultados del trabajo académico: "justamente el grupo donde han colaborado de forma regular las familias, el progreso ha sido mejor. Se ayudan más, hay más compañerismo, han superado más retos que el otro curso [con menor regularidad en la participación]. Y es una realidad" (GD, 691-693).

A su vez, la participación de familiares permite una reorganización de los recursos humanos para atender más eficazmente las necesidades del alumnado. La presencia de más personas adultas interaccionando con los niños y niñas posibilita al profesorado mejores condiciones y más tiempos de calidad para atender las necesidades del alumnado dentro de un marco común de trabajo dentro de la filosofía inclusiva (Booth \& Ainscow, 2015): "el hecho de que haya familias te ayuda a que demos más apoyo a quien más lo necesite" (GD, 384-385) y, en la línea, "porque si estabas tú solo no 


\section{Castanedo \& Capllonch - Families involvement in P. E.}

tenías tiempo para todo, entonces, pues las familias nos pueden ir ayudando" (RA7, 10-11).

Este apoyo se vuelve fundamental para el alumnado más vulnerable (Fitch, 2003), evidenciando que la participación de familiares favorece la implicación de niños y niñas con mayores dificultades motrices o de relaciones sociales y facilita avanzar hacia la igualdad de las diferencias (Aubert et al, 2008), es decir, hacia una generalización de los mejores resultados posibles para todos y todas siendo conscientes de las diferencias y dificultades existentes: "llegó un momento en el que no había diferencias casi, siempre con apoyos, con apoyo de las familias" (RF5, 96-97), ya que los apoyos se focalizaban en quienes más los necesitaban: "he ayudado a todos los que he visto que lo necesitaban (...) Lo mismo que ayudas a tu hija, pues intentas ayudar a todos los que tienen dificultad " (RF3, 247, 251252).

Como señala Flecha (2012), cuando se organiza el aprendizaje en base a los principios del ApD, que fundamentan el modelo RIRC, y se incluyen interacciones con personas adultas que no son docentes, el alumnado aprende más y mejor en un entorno más acorde con la actual sociedad de la información. Los aprendizajes derivados de las interacciones con familias diversas se traducen en mejoras en los resultados curriculares y convivenciales, en línea con los resultados obtenidos en el Proyecto INLUD-ED Consortium (2011) o en el estudio longitudinal de Dearing et al. (2006). Los resultados curriculares mejoraron significativamente en relación a los periodos previos donde no participaron las familias, sobretodo en aquellos grupos donde el voluntariado participó de una manera más regular, estable, consciente y activa: "empezamos a venir y la evolución era totalmente diferente. Al principio había algún reto que se superaba [y, a partir de ahí, los empezaron] a superar todos o, bueno, la gran mayoría" (RF4, 194-197). Este avance también era percibido por el alumnado: "hemos aprendido mucho: a comportarnos, a trabajar en grupo y, sobre todo, con los familiares hemos aprendido muchísimo" (RA7, 4-6).

Se destaca especialmente en los relatos la mejora de todos y todas sin que nadie quedara excluido: "ha sido un sistema distinto y yo, para mí, ha sido como que mejoraban todos en todo (...), veías la mejoría, o sea, de clase en clase" (RF2, 380-381, 386). Así, se ha evidenciado que se incrementan los resultados de todos y todas, incluido el alumnado con mayores dificultades o barreras motrices y/o de relaciones sociales: "en el 
primer trimestre solo superé dos o tres retos y en los dos últimos trimestres superé todos" (RA1, 21-23) o "N. ha superado todos los retos antes que gente que antes superaba los retos con anterioridad (...) y A. lo mismo" (RF1, 280-281, 283).

En relación a la mejora de la convivencia y la cohesión grupal, la progresión también se ha visto en el alumnado con baja integración en el grupo, apreciándose una mejor relación con sus compañeros y compañeras. Lejos de ser casos aislados, reflejan la dinámica general:

estas clases le han venido súper bien para integrarse más, estar más con todos, porque todos también han tenido que ayudarle a ella muchas veces. $\mathrm{Y}$ entonces, cuando le han ayudado ella se ha sentido... Venía a casa, bueno, pero emocionada, es que emocionada (RF, 87-89, 91).

En la línea, cabe destacar dos elementos: a) el aprendizaje y el sentimiento de competencia de alumnado, y b) el diálogo como herramienta para gestionar conflictos: "conflictos así muy suaves que entre ellos mismos y entre los compañeros (...) los debatían” (RF1, 593-594). La combinación de diálogo y aprendizaje favoreció un ambiente de trabajo y respeto: "no he visto a nadie que se ría de nadie porque no le sale [el reto]" (RF3, 309-310). Las familias han sido un factor clave en la articulación de estos dos elementos. Como se ha evidenciado anteriormente, la participación de familiares facilita más y mejores aprendizajes permitiendo atender a más alumnado a la vez y más eficazmente; y, por otro lado, porque la dinamización de las interacciones se produce a través de diálogos con personas diversas. Además, este voluntariado actúa como elemento cohesionador del grupo: "yo aportaba como más, no sé, sensación de grupo (...) Se notaba además mucho. Eso sí que me gustaba porque al final lo notas" (RF2, 343-344, 485-486); y su implicación hacía sentirse y comportarse mejor a la mayoría de los niños y niñas. El hecho de contar con más recursos humanos asegura más apoyos necesarios y brinda la oportunidad al profesorado de centrarse en aspectos relacionados con la gestión de conflictos, un ámbito clave también para el aprendizaje y la convivencia: 
el hecho de que haya tantas personas adultas dentro del aula hace que se pueda gestionar muchísimo más fácilmente cualquier conflicto. Que no pase desapercibida una falta de respeto que no tenga respuesta. Entonces eso, claro, modifica el clima del aula completamente (GD, 663-665).

Además de la mejora curricular y convivencial, se ha evidenciado el aumento de la autoestima, la confianza y la seguridad del alumnado, principalmente en aquel con mayores dificultades: "hay niños que enseguida los superaban [los retos], otros que les costaba más, pero al final lo acababan haciendo. Sobretodo esos niños que les costaba hacerlo se han dado cuenta que pueden hacer lo mismo que el resto" (RF4, 203-205); una realidad gracias a que "el apoyo de las familias ha ayudado a niñas que a lo mejor en un principio las familias, el centro, los daban ya desahuciados en cuanto a EF" (GD, 446-448). El alumnado, así, interacciona con personas que creen en sus capacidades y posibilidades, lo que genera un aumento de las expectativas y un mayor bienestar y confianza: "ayudar, ayudabas a todos, incluso había niños que cuando te veían que estabas se alegraban como si hubiera venido... ¡lo mismo que veías que se alegraba tu hijo!” (RF4, 87-88). Saber que había familiares generaba seguridad en algunos niños y niñas al tener la posibilidad de contar con su ayuda en cualquier momento: "más seguridad de decir: 'pues bueno, si mi madre viene aquí es que me va a ayudar y ayuda también a mis compañeros" (RF3, 333-334). Coincidiendo con Aubert et al. (2008), se constata que las interacciones que aumentan los aprendizajes y el sentido de aprender generan a su vez aumento de la autoestima, la confianza y la seguridad del alumnado.

Otro resultado derivado de esta investigación y acorde con las evidencias presentadas en el estudio europeo sobre clima escolar (Gázquez, Pérez, \& Carrión, 2011), es la existencia de una asociación entre la participación de familiares y el aumento del nivel de motivación del alumnado. La presencia de familiares genera en la mayoría de los niños y niñas una alta motivación, ilusión y ganas de demostrar lo que son capaces de hacer, superándose y esforzándose en cada sesión: "se subió mucho el compañerismo, el nivel de los niños, la motivación. Estaban súper motivados, estaban muy motivados" (RF5, 270-271). Aunque en un principio podía percibirse como una motivación extrínseca únicamente condicionada por la presencia de familiares, "al final les hace creer en ellos 
mismos y ver que lo pueden conseguir" (RF3, 117-118). Además, se evidenció que el incremento de la motivación se trasladaba fuera del horario de clase y fuera del centro escolar. El horario de recreos, establecido como tiempo para la ampliación del aprendizaje y mejora de los resultados (Valls \& Siles, 2012), era aprovechado voluntariamente por la mayor parte del alumnado para el trabajo y la práctica de retos. Varios niños y niñas, además, solicitaban material de EF para utilizarlo en la calle y trabajar fuera del horario lectivo en los retos establecidos. Los aprendizajes se transferían además a otros espacios, transformándose el ámbito comunitario al tornarse habitual ver a niños y niñas del colegio jugando en la plaza con combas, palas, peonzas o patinetes; situaciones que con anterioridad no se producían y que modificaban también la comunicación en el entorno familiar: "se nota que están muy motivados y no solo en clase porque en casa $\mathrm{N}$. está todo el día hablando de los retos que tiene conseguidos, de lo que queda..." (OPc, 269-271). No solo la motivación trascendió el espacio del aula, sino que las ayudas de familiares traspasaron el espacio y tiempo de las sesiones de EF. Dado que los familiares que participan en las clases saben qué hay que hacer y cómo para superar un reto, se posibilita que las ayudas puedan continuar en contextos diferentes al escolar. Varios testimonios así lo corroboran, como el de una madre que comentaba que iba a comprar palas para practicar con su hija por las tardes (OPc, 39-40), otra familia que explicaba que habían comprado material porque estaban trabajando con su hija en casa (OPc, 54-56) o un padre que manifestó en una sesión que "los malabares los puede hacer en casa. Yo le ayudo" (OPc, 132), aprovechando el tiempo de clase para otro reto.

Por otro lado, las familias son un referente de solidaridad para el alumnado. Se convierten en un modelo adulto diferente al profesorado que contribuye al principio de inteligencia cultural (Oliver, Botton, Soler, \& Merrill, 2011), como refleja un padre: "que pueden [el alumnado] aprender de gente que no sea solo, por ejemplo, un profesor" (RF2, 364-365). Además, transmiten y demuestran acciones y valores socialmente aceptados como el esfuerzo, la superación, la ayuda o sentimientos como la solidaridad al colaborar desinteresada y voluntariamente con el objetivo de que todos los niños y niñas aprendan más. 


\section{Dimensión Transformadora: Aportación a las Familias. Situaciones, Hechos o Actuaciones en las que la Participación de las Familias les Facilita o Posibilita Aprendizajes}

La participación de familiares posibilita establecer diálogos transformadores. Estar en el aula aporta a las familias información muy importante para transformar ideas previas sobre aspectos relacionados con la educación de sus hijos o hijas. Muchas madres, después de dialogar y ver lo que sus hijos e hijas eran capaces de hacer, se daban cuenta de que les habían educado en el miedo constante limitando sus experiencias motrices (OPc, 16-20) o de que habían infravalorado las posibilidades de su hija: "pensaba que T. había muchas cosas que no las iba a poder conseguir y yo misma me he sorprendido" (RF3, 118-119).

La participación educativa de las familias posibilita conocer el mundo de la escuela en el que viven sus niños y niñas. Conocen qué hacen en el día a día, cómo y con quién se relacionan o cómo se comportan. Un padre comentaba en relación a su hija: "no sabía lo que hacía en el colegio y ahora sí (...) Aquí me entero de todo, claro" (RF2, 152-153, 155). Una madre señalaba con gran sinceridad: "yo lo que quería era ver el comportamiento de ella [su hija] en clase y ver cómo se relacionaba con los demás. Pues me ha aportado otra visión" (RF3, 84-85). Todas las familias agradecen la posibilidad de ver lo que hacen sus niños y niñas en el entorno escolar y de formar parte de él, valorándolo como una oportunidad que también refuerza el vínculo familia-escuela y contribuye a revalorizar la labor docente y el sentimiento de que el profesorado trabaja para la mejor educación para sus hijos e hijas:

¡cómo los tratáis, cómo los implicáis en los retos, cómo los ayudáis, es que hasta con psicología! Vamos, es que me ha impresionado, os lo juro (...) Es muy de agradecer vivirlo, porque si no lo vives te crees que un profesor no hace eso (GD, 513-514, $520)$.

La participación de familias facilita crear más y mejores apoyos dentro y fuera de clase, aportando información y formación sobre la mejor manera de ayudar a sus hijos e hijas (OPc, 46-48) y con el objetivo de mejorar sus resultados: "para ayudar a mi hija porque la veía que no..., y lo pasaba mal. 
Mamá que no puedo, que no puedo. Y lo intentábamos fuera de clase" (RF1, 160-161).

La participación regular y activa de las familias favorece su implicación en el entorno escolar y genera sentimientos de pertenencia a la comunidad educativa. El voluntariado se sentía útil (RF3, 180-181, 294), parte importante del grupo (RF1, 452-453) y aumentaba su bienestar: "[me lo he pasado] iigenial!!, he vuelto a mi niñez" (RF1, 15) o "he estado cómodo, a gusto, he disfrutado" (RF4, 29). Las familias intervenían en las evaluaciones diarias y aportaban argumentos al profesorado en relación a aspectos de la organización de la clase como los agrupamientos o las valoraciones individuales del trabajo del alumnado (GD, 812-813). El hecho de que se sintieran a gusto, importantes y útiles, ayudaba a crear sentido de pertenencia en muchas de las personas: "es que me sentía fenomenal. Iba a trabajar y a todo correr, yo digo, aunque sea veinte minutos para darle ahí ánimo cuando faltaba solo un reto, o dos" (RF1, 245246) o "me iba integrando cada vez más, veía que cada vez me gustaba más ayudarles" (RF3, 184).

Ni todos los tipos de participación generan el mismo impacto (Boonk, Gijselaers, Ritzen, \& Brand-Gruwel, 2018), pudiendo incluso establecerse relaciones negativas o indeseadas (Fan \& Chen, 2001; Girbés et al., 2015), ni todas las formas de gestionar el aula y el aprendizaje son eficaces (American Psychological Association, 2015). La gestión eficaz del aula y del aprendizaje en el modelo RIRC favorece que los familiares quieran volver a participar. Un padre comentaba la diferencia con otro sistema de participación educativa de familiares con el que no había experimentado tanta fidelidad y eficacia: "ha sido el sistema distinto y yo para mí ha sido como que mejoraban todos en todo (...). Las pautas a seguir [por el alumnado] eran..., pues perfectas" (RF2, 380-381, 412-413). En este sentido, son varios los relatos que hacen referencia a la organización y gestión del aprendizaje en el modelo RIRC y a la constatación de resultados de aprendizaje para todo el alumnado como motivos para continuar participando (RF3, 451-452, 454; OPc, 89-90; OPc, 136). 


\section{Dimensión Exclusora: Aportación de las Familias. Situaciones, Hechos o Actuaciones Derivadas de la Participación de Familiares que Impiden o Limitan Aprendizajes}

La pasividad en la participación incide en la calidad y cantidad de las interacciones con el alumnado. Escasos y excepcionales fueron los casos de familiares que mantuvieron una actitud pasiva durante todas las participaciones, como refleja este testimonio: "siempre me he mantenido más precavida, ¿sabes? (...) Me he mantenido más prudente porque no quería yo intervenir en nada, o fastidiarla (...) Activa físicamente pero igual pasiva en motivación" (RF5, 110-112, 116). Lo que sí era habitual en la primera o primeras sesiones era que se mostraran expectantes, con una actitud menos activa y de mera observación: "al principio estaban en una posición más esperando" (RF3, 188-189). En sesiones sucesivas, el voluntariado generaba confianza y mostraba actitudes más activas, dinamizadoras y comunicativas con el alumnado.

La falta de regularidad en la participación no favorece la creación de sentido. Una alumna comentaba que "con una vez no basta, con una vez has visto; si haces más veces ya comprendes la colaboración, el comportamiento de los demás, todo" (GD, 74-75), lo que era corroborado por familias de participación intensa: "el problema es que hay mucha gente que ha dicho: 'no, yo ya he ido una vez, ya lo he visto y ya he cumplido'" (GD, 70-71).

Además, la creencia de que un hijo o hija no necesita ayuda limita la participación de las familias. Pensar que un hijo o hija no necesita ayuda en EF o en cualquier otra materia, limita la participación educativa de las familias. Algunos familiares han asistido a una sesión de EF y no han vuelto: "hay gente que comentaba que igual hay familias que venían para ver simplemente y como sabían que sus hijos no necesitaban ayuda pues no volvían" (RF3, 211-212), "hay muchos que no participan en realidad porque piensan que no es necesario" (RF2, 75). Para tener en cuenta en sucesivas experiencias, estas actitudes y pensamientos limitantes pueden transformarse aportando por un lado evidencias científicas que vinculan la implicación familiar y el aprendizaje de todos los niños y niñas (INCLUDED Consortium, 2011) y, por otro, trasmitiendo el concepto de "egoísmo inteligente", pues cuantas más interacciones de calidad existan entre las personas que forman un grupo, mayores aprendizajes se producirán también 
a nivel individual. Como señalan Aubert et al. (2008), “cuando el egoísmo tiene efectos positivos en otras personas y no solo en nosotros y nosotras (...), puede ser deseable" (p. 227).

En relación a las interacciones de calidad generadoras de aprendizaje, es importante asegurar la estabilidad en la asistencia debido a que más participantes no es sinónimo de mejores resultados. El grupo de $3^{\circ}$ es un ejemplo claro: en el segundo trimestre participaron 17 familiares y en el tercer trimestre 10 , siendo los resultados de retos superados prácticamente los mismos $-92 \%$ en el segundo trimestre y $93 \%$ en el tercero-. Sin embargo, en ambos trimestres dos familiares asistían regularmente. Las interacciones de calidad son sinónimo de aprendizaje y la regularidad en la participación facilita la calidad en las interacciones.

\section{Dimensión Exclusora: Aportación a las Familias. Situaciones, Hechos o Actuaciones Derivadas de la Participación de Familiares que les Impiden o Limitan Aprendizajes}

Algunos aspectos fácilmente transformables en próximas experiencias han podido limitar la participación de familiares. Por un lado, la falta de difusión por parte de las escuelas limita el conocimiento de la importancia de la participación educativa y evaluativa de familiares. Por otro lado, la relación tradicional escuela-familia se identifica como factor limitante de la participación, ya que "siempre que vienes al colegio es para algo malo, casi siempre; o ha tenido un accidente, o se ha puesto malo, o ha tenido una bronca con otro niño..." (RF1, 74-76). En este sentido, las familias señalan que nunca antes se les había invitado a participar de manera educativa, partiendo de un desconocimiento absoluto que generaba, sobre todo, mucha inseguridad: "nunca se había ofrecido una cosa así" (RF3, 70), "es la primera vez. Nunca nos habían invitado" (RF5, 58). La incertidumbre de no saber cómo se va a desarrollar la participación o cómo se van a establecer las relaciones con el profesorado pueden condicionar la decisión de participar: "sí sabes más o menos para qué vienes, pero tampoco sabes cómo va a ir en el sitio" (RF2, 56-57). La incertidumbre, las inseguridades, la tradicional relación jerárquica impuesta por la escuela con respecto a las familias, la percepción de incompetencia, la exposición personal en un entorno desconocido o los estereotipos de género son factores que algunas madres han identificado como limitantes. 
No percibir que la participación familiar tiene relevancia para el aprendizaje de los niños y niñas es un factor limitante de la participación. A pesar de que parece haber consenso en la claridad de la carta informativa inicial, hasta que no se participa y se vivencia la dinámica de las clases no se es consciente del papel a desempeñar y de los beneficios que aporta la participación educativa de familiares: "sí, bueno, sí, quedaba claro pero luego cuando ves las clase es realmente cuando te das cuenta de todos los aspectos que se tocan y cómo se trabaja" (RF3, 16-17); "yo creo que aunque sí se sabía lo que se venía a hacer, hasta que no lo vives no sabes exactamente lo que es" (RF4, 19, 20). Además de reuniones informativas, de coordinación y formativas con el voluntariado, varios testimonios proponen la difusión de los beneficios de la participación de familiares a través de recursos como el blog del centro con vídeos, entrevistas o testimonios para hacer llegar la información desde distintos canales y fomentar la participación (GD).

\section{Conclusiones}

En una de las primeras referencias que instaban a la promoción de la participación de familias en EF, Wilcox (1988) ya indicaba el gran perjuicio que implicaba la decisión de aislar a las familias del contexto escolar y de la EF concretamente. Tres décadas después, las experiencias más actuales de participación educativa de familias en el área de EF registradas a nivel internacional (Allar, Elliott, Jones, Kristjansson, Taliaferro, \& Bulger 2017; Bodnarchuk, Rymar, Solovey, \& Malanchuk, 2018; Hager, \& Beighle, 2006) y a nivel nacional (Capllonch, 2008-2011; Capllonch, Figueras, \& Castro, 2018; Castro, Gómez, \& Macazaga, 2014; Andrés Fabra, 2017) son escasas pero alentadoras.

La investigación presentada se ha desarrollado en torno a la AEE "participación educativa de la comunidad", actuación que ha demostrado generar los mejores resultados en diferentes contextos socioeducativos (American Psychological Association, 2015; Díez et al., 2011; INCLUDED Project, 2006-2011). Aunque su implementación se haya centrado en EF se han obtenido resultados muy similares a los descritos en el proyecto INCLUD-ED Consortium (2011), que se centraba en áreas instrumentales, en relación a la mejora de los resultados de todo el alumnado, la mejora de la convivencia del grupo y el aumento de valores y sentimientos deseables. 
Este trabajo aporta más evidencias acerca de que la participación educativa de familias dentro de los centros educativos, y concretamente en el área de $\mathrm{EF}$, es especialmente decisiva en el aprendizaje y relaciones sociales del alumnado con más barreras o dificultades de aprendizaje. Entre otras, se pueden destacar las siguientes evidencias: un incremento de las interacciones comunicativas, un incremento de la implicación de niños y niñas con mayores dificultades motrices o de relaciones sociales, aumento del sentimiento de competencia del alumnado, aumento de la motivación y mejora de las relaciones entre compañeros y compañeras.

Se contribuye aportando evidencias desde el área de EF para reforzar un vínculo dialógico entre escuela y familia, por ser dos de las instituciones con mayor responsabilidad compartida en la educación de los niños y niñas. La participación de familiares ha facilitado en primer lugar un cambio en la concepción de la EF, valorando el potencial educativo del área. Por otro lado, ha posibilitado el aumento de los factores sobre los que hay un acuerdo unánime de sus efectos positivos (Boonk et al, 2018): altas expectativas sobre los niños y niñas, hablar de la escuela en casa, aumento de la comunicación maestro-familias, aumento de apoyo al aprendizaje tanto en clase como fuera del horario lectivo (practicando retos en el parque, comprando material para poder practicar en la calle, etc.). También fue de gran importancia el hecho de que los familiares percibiesen, de sesión en sesión, que su presencia tenía relevancia directa en sus hijos o hijas, o en otros niños y niñas. Esto favorecía una mayor participación y generaba una creación de sentido con la implicación en la escuela; se producía una fidelización. Esta relación escuela-familia es clave porque mejora la coordinación de las interacciones de los niños y niñas (Aubert et al, 2008), lo que impacta positivamente en su desarrollo y aprendizaje (Díez et al, 2011). Los resultados sugieren la importancia de crear en los centros educativos espacios de aprendizaje donde los niños y niñas puedan interaccionar con familiares y personas de su contexto para unir la escuela, la vida comunitaria, el currículum y sus identidades, y de esta manera desarrollar la creación de sentido en el alumnado y sus familias.

No obstante, a pesar de la extensa literatura científica que evidencia la importancia de la participación educativa de las familias en la educación de sus hijos e hijas, son necesarias nuevas líneas de investigación desde el área de Educación Física. Proponemos, por un lado, romper las barreras detectadas que dificultan la participación familiar y que hemos identificado 
en las creencias por parte de algunos familiares de la escasa necesidad o importancia de ayudar en la escuela, la escasa o nula información que la escuela aporta a las familias sobre los beneficios y los tipos de participación, y la relación tradicional y jerárquica que impone la escuela relegando a las familias a la mera información o consulta. Por otro lado, se precisa de más investigación para evidenciar cómo desde el área de EF es posible contribuir, con la colaboración de las familias y de la comunidad, a la superación de desigualdades por razones culturales, a la superación de desigualdades de género, a la creación de modelos de género alternativos y al desarrollo de relaciones más igualitarias.

\section{Notas}

${ }^{1}$ A.= apoyo; I.= información; P.= participación; R.= resultados; $\mathrm{T} .=$ transferencia; $\mathrm{VyS}=$ valores y sentimientos.

\section{References}

Alexiu, T., \& Sordé T. (2011). How to turn difficulties into opportunities: Drawing from diversity to promote social cohesion. International Studies in Sociology of Education, 21(1), 49, 62. doi:10.1080/09620214.2011.543852

Allar, I., Elliott, E., Jones, E., Kristjansson, A. L., Taliaferro, A., \& Bulger, S. M. (2017). Involving families and communities in CSPAP development using asset mapping. Journal of Physical Education, Recreation \& Dance, 88(5), 7-14. doi:10.1080/07303084.2017.1280439

An, J. H., \& Hodge, S. R. (2013). Exploring the Meaning of Parental Involvement in Physical Education for Students with Developmental Disabilities. Adapted Physical Activity Quarterly, 30(2), 147-163. doi:10.1123/apaq.30.2.147

American Psychological Association, Coalition for Psychology in Schools and Education (2015). Top 20 principles from psychology for preK12 teaching and learning. Recuperado de http://www.apa.org/ed/schools/cpse/top-twenty-principles.pdf 
Andrés Fabra, J. A. (2017). Fomento de práctica físico-deportiva desde el contexto escolar a través del aprendizaje dialógico. (Tesis doctoral) Universidad Miguel Hernández, Elche, España.

Apple, M. W., \& Beane, J. A. (2012). Escuelas democráticas (5ª ed.). Madrid: Morata.

Aubert, A., Flecha, A., García, C., Flecha, R., \& Racionero, S. (2008). Aprendizaje dialógico en la sociedad de la información. Barcelona: Hipatia.

Bodnarchuk, O., Rymar, O., Solovey, A., \& Malanchuk, H. (2018). The interaction of school and family in physical education of first grade students. Journal of Physical Education and Sport, 18, 1092-1098. doi:10.7752/jpes.2018.s2163

Bonell, L., \& Ríos, O. (2014). Participation and family education in school: Successful educational actions. Studies in the Education of Adults, 46(2), 177-191. doi:10.1080/02660830.2014.11661665

Boonk, L., Gijselaers, H. J. M., Ritzen, H., \& Brand-Gruwel, S. (2018). A review of the relationship between parental involvement indicators and academic achievement. Educational Research Review, 24, 10-30. doi:10.1016/j.edurev.2018.02.001

Booth, T., \& Ainscow, M. (2015). Guía para la evaluación y mejora de la educación inclusiva: desarrollando el aprendizaje y la participación en los centros escolares. Madrid: Consorcio para la Educación Inclusiva y FUHEM. Recuperado de http://www.oei.es/noticias/spip.php?article15212

Capllonch, M. (2008-2011). Juega Dialoga y Resuelve. La superación de conflictos en educación física mediante el modelo comunitario. Diseño de un programa específico para comunidades de aprendizaje, con referencia, SEJ2007-61757/EDUC. Plan Nacional I+D+I.

Capllonch Bujosa, M., Figueras, S., \& Castro, M. (2018). Conflict Resolution Strategies in Physical Education. Apunts Educacion Fisica y Deportes (133), 50-67. doi:10.5672/apunts.20140983.es.(2018/3).133.04

Caspe, M., Lopez, M., \& Wolos, C. (2007). Family involvement makes a difference. 2, Winter 2006/2007. Cambridge: Harvard Family Research Project, Harvard Graduate School of Education.

Castanedo, J. M., \& Capllonch, M. (2017). Evaluación compartida y formativa en el modelo inclusivo retos individuales con 
330 Castanedo \& Capllonch-Families involvement in P. E.

responsabilidad compartida. Infancia, Educación y Aprendizaje, 3(2), 118-126. doi:10.22370/ieya.2017.3.2.710

Castro, M., Gómez, A., \& Macazaga, A. Ma . (2014). Aprendizaje dialógico y grupos interactivos en educación Física. Retos. Nuevas tendencias en Educación Física, Deporte y Recreación, 25, 174-179.

Recuperado de http://retos.org/numero_25/25-35.html

Dearing, E., Kreider, H., Simkins, S., \& Weiss, H. B. (2006). Family involvement in school and low-income children's literacy performance: Longitudinal associations between and within families. Journal of Educational Psychology, 98(4), 653-664.

doi:10.1037/0022-0663.98.4.653

Driessen, G., Smit, F., \& Sleegers, P. (2005). Parental involvement and educational achievement. British Educational Research Journal, 31(4), 509-532. doi:10.1080/01411920500148713

Díez, J., Gatt, S., \& Racionero, S. (2011). Placing immigrant and minority family and community members at the school's centre: The role of community participation. European Journal of Education, 46(2), 184-196. doi:10.1111/j.1465-3435.2011.01474.x

Erwin, H., Beets, M. W., Centeio, E., \& Morrow, J. R. (2014). Best practices and recommendations for increasing physical activity in youth. Journal of Physical Education, Recreation \& Dance, 85(7), 27-34. doi:10.1080/07303084.2014.937197

Fan, X., \& Chen, M. (2001). Parental involvement and students' academic achievement: A metaanalysis. Educational Psychology Review, 13, 1-22. doi:10.1023/A:100904881

Fitch, F. (2003). Inclusion, Exclusion, and Ideology: Special Education Students' Changing Sense of Self. The Urban Review, 35(3), 233252. doi:10.1023/A:1025733719935

Flecha, A. (2012). Family education improve student's academic performance: Contributions from european research.

Multidisciplinary Journal of Educational Research, 3, 301-321. doi:10.4471/remie

Flecha, A., García, R., Gómez, A., \& Latorre, A. (2009). Participación en escuelas de éxito: Una investigación comunicativa del proyecto Includ-ed. Cultura y Educación, 21(2), 183-196. doi:10.1174/113564009788345899 
Flecha, R. (1997). Compartiendo palabras: El aprendizaje de las personas a través del diálogo. Barcelona: Paidós.

Flecha, R. (2011). The dialogic sociology of education. International studies in sociology of education, 21(1), 7-20. doi:10.1080/09620214.2011.543849

Flecha, R., \& Soler M. (2013). Turning difficulties into possibilities: engaging Roma families and students in school through dialogic learning. Cambridge Journal of Education, 43(4), 451-465. doi:10.1080/0305764X.2013.819068

Garbacz, S. A., Zerr, A. A., Dishion, T. J., Seeley, J. R., \& Stormshak, E. (2018). Parent Educational Involvement in Middle School: Longitudinal Influences on Student Outcomes. Journal of Early Adolescence, 38(5), 629-660. doi:10.1177/0272431616687670

García, R. (2012). Out of the ghetto psychological basis of dialogic learning. International Journal of Educational Psychology, 1, 51-69. doi:10.4471/ijep.2012.04

Gatt, S., Ojala, M., \& Soler, M. (2011). Promoting social inclusion counting with everyone: Learning Communities and INCLUD-ED. International Studies in Sociology of Education, 21(1), 33-47. doi:10.1080/09620214.2011.543851

Gázquez, J. J., Pérez M. M., \& Carrión, J. J. (2011). Clima escolar y resolución de conflictos según el alumnado: un estudio europeo. Revista de Psicodidáctica, 16(1), 39-58. doi:10.1387/RevPsicodidact.1144

Girbés, S., Macías, F., \& Álvarez, P. (2015). De la escuela gueto a una comunidad de aprendizaje: Un Estudio de caso sobre la superación de la pobreza a través de una educación de éxito. International and Multidisciplinary Journal of Social Sciences, 4(1), 88-116. doi:10.17583/rimcis.2015.04

Gokturk, S., \& Dinckal, S. (2017). Effective parental involvement in education: experiences and perceptions of Turkish teachers from private schools. Teachers and Teaching, 24(2), 183-201. doi:10.1080/13540602.2017.1388777

Gómez, A., Puigvert, L., \& Flecha, R. (2011). Critical communicative methodology: Informing real social transformation through research. Qualitative Inquiry 17(3), 235-245. doi:10.1177/1077800410397802 
Gómez, A., Racionero, S., \& Sordé, T. (2010). Ten years of critical communicative methodology. International Review of qualitative Research, 3(1), 17-43. doi:10.1525/irqr.2010.3.1.17

Gómez, J., Latorre, A., Sánchez, M., \& Flecha, R. (2006). Metodología comunicativa crítica. Barcelona: Paidós.

Hager, L., \& Beighle, A. (2006). Promoting physical activity through physical education: Increasing parental involvement. Teaching Elementary Physical Education, 17(1), 28-31.

Hampden-Thompson, G., \& Galindo, C. (2017). School-family relationships, school satisfaction and the academic achievement of young people. Educational Review, 69(2), 248-265. doi:10.1080/00131911.2016.1207613

Henderson, A. T., \& Mapp, K. L. (2002). A new wave of evidence: The impact of school, family and community connections on student achievement. Annual synthesis. Washington, DC: National Centre for Family y Community Connections with Schools, Institute of Education Sciences.

Hill, N. E., \& Taylor, L. C. (2004). Parental school involvement and children's academic achievement. Pragmatics and issues. Current Directions in Psychological Science, 13(4), 161-164. doi:10.1111/j.0963-7214.2004.00298.x

INCLUD-ED Project. Strategies for inclusion and social cohesion in Europe from education. (2006-2011). 6th Framework Programme. Citizens and Governance in a Knowledge-based Society. CIT4-CT2006-028603. Directorate General for Research, European Commission. Recuperado de http://creaub.info/included/ INCLUD-ED Consortium. (2011). Actuaciones de éxito en las escuelas europeas. Madrid: Instituto de Formación del Profesorado, Investigación e Innovación Educativa (IFIIE), Ministerio de Educación. Recuperado de https://sede.educacion.gob.es/publiventa/PdfServlet?pdf=VP14963.p df\&area $=\mathrm{E}$

Kehm, R., Davey, C. S., \& Nanney, M. S. (2015). The Role of Family and Community Involvement in the Development and Implementation of School Nutrition and Physical Activity Policy. Journal of School Health, 85(2), 90-99. doi:10.1111/josh.12231 
Lincoln, Y., \& Guba, E. (1985). Naturalistic Inquiry. Beverly Hills, C.A.: Sage.

Ma, X., Shen, J. P., Krenn, H. Y., Hu, S. S., \& Yuan, J. (2016). A MetaAnalysis of the Relationship Between Learning Outcomes and Parental Involvement During Early Childhood Education and Early Elementary Education. Educational Psychology Review, 28(4), 771801. doi:10.1007/s10648-015-9351-1

Martínez, B., \& Niemelä, R. (2010). Formas de implicación de las familias y de la comunidad hacia el éxito educativo. Revista Educación y Pedagogía, 56, 69-77. Recuperado de http://aprendeenlinea.udea.edu.co/revistas/index.php/revistaeyp/articl e/view/9821

Mercer, N., Hargreaves, L., \& García, R. (2017). Aprendizaje e interacciones en el aula. Barcelona: Hipatia.

Michael, S., Dittus, P., \& Epstein, J. (2007). Family and community involvement in schools: results from the School Health Policies and Programs Study 2006. Journal of School Health, 77(8), 567-587. doi:10.1111/j.1746-1561.2007.00236.x

Oliver, E., de Botton, L., Soler, M., \& Merrill, B. (2011). Cultural Intelligence to Overcome Educational Exclusion. Qualitative Inquiry, 17(3), 267-276. doi:10.1177/1077800410397805

Pomerantz, E., Grolnick, W. S., \& Price, C. E. (2005). The role of parents in how children approach school: a dynamic process perspective. En J. Elliot \& C.S. Deck (Eds.), The handbook of competence and motivation, (pp. 259-78). New York: Guilford.

Puigvert, L., Christou, M., \& Holford, J. (2012). Critical communicative methodology: Including vulnerable voices in research through dialogue. Cambridge Journal of Education, 42, 513-526. doi:10.1080/0305764X.2012.733341

Racionero, S. (2010). Egalitarian dialogue and instrumental dimension. Two principles of dialogic learning in the classroom. Psychology, Society \& Education, 2(1), 61-70. doi:10.25115/psye.v2i1.436 Racionero, S., Ortega, S., García, R., \& Flecha, R. (2012). Aprendiendo contigo. Barcelona: Hipatia.

Sénéchal, M., \& LeFevre, J. (2002). Parental involvement in the development of children's reading skill: A five-year longitudinal 
334 Castanedo \& Capllonch-Families involvement in P. E.

study. Child Development, 73(2), 445-460. doi:10.1111/14678624.00417

Sordé, T., \& Ojala, M. (2010). Actos comunicativos dialógicos y actos comunicativos de poder en la investigación. Revista Signos, 43(2), 377-391. doi:10.4067/S0718-09342010000400008

Tellado, I., \& Sava, S. (2010). The role of non-expert adult guidance in the dialogic construction of knowledge. Revista de Psicodidáctica, 15(2), 163-176. Recuperado de http://www.redalyc.org/html/175/17517246002/

Valls, R., \& Siles, G. (2012). L'ampliació del temps d'aprenentatge, una pràctica inclusiva que millora el rendiment acadèmic de tot l'alumnat. Pedagogia i Treball Social. Revista de Ciències Socials Aplicades, 2(1), 46-62. Recuperado de https://www.raco.cat/index.php/PiTS/article/view/264884

Vygotsky, L. (1979). El desarrollo de los procesos psicológicos superiores. Barcelona: Crítica.

Wilhelmsen, T., \& Sørensen, M. (2018). Physical education-related homeschool collaboration. European Physical Education Review, 1-17. doi:10.1177/1356336x18777263

Wilkos, R. C. (1988). Promoting parents as partners in physical education. Physical Educator, 45(1), 19-23. Recuperado de https://search.proquest.com/openview/dec22d15d56d2f51cd17c90d6 b40c28b/1?cbl=2030480\&pq-origsite $=$ gscholar

José Manuel Castanedo is PhD Student at the Faculty of Education at University of Barcelona, Spain.

Marta Capllonch is Senior Lecturer at the Faculty of Education at University of Barcelona, Spain.

Contact Address: José Manuel Castanedo, University of Barcelona, Faculty of Education, Pg. de la Vall d'Hebron, 171. CP. 08035, Barcelona, España. Email: jcastaal7@alumnes.ub.edu 